\section{Precipitation of hepatic encephalopathy by propranolol in cirrhosis}

Continuous oral propranolol reduces portal venous pressure in patients with cirrhosis. ${ }^{1}$ A controlled trial in such patients who had bled from varices showed a significantly lower incidence of rebleeding in those given propranolol." This led to the widespread and enthusiastic use of propranolol to prevent recurrent variceal haemorrhage in patients with cirrhosis. The beneficial effect of propranolol, however, has so far been demonstrated only in predominantly alcoholic patients who were all in good condition and in whom side effects of propranolol were few. It is not known whether prophylaxis with propranolol reduces the incidence of variceal bleeding in patients with other causes of cirrhosis or more severe liver failure. Complications of treatment with propranolol may be more common in these patients. We report on a patient in whom propranolol given to prevent recurrent variceal bleeding precipitated hepatic encephalopathy.

\section{Case report}

A 69 year old woman presented with bleeding oesophageal varices in 1979. This settled spontaneously without active treatment. She suffered from cryptogenic cirrhosis and insulin dependent diabetes mellitus but remained well until June 1982, when she had a further variceal haemorrhage. This settled after blood transfusion alone.

On admission the severity of her liver dysfunction was assessed as Child's grade A. Serum bilirubin concentration was $13 \mu \mathrm{mol} / \mathrm{l}(760 \mu \mathrm{g} / 100 \mathrm{ml})$, serum albumin concentration $37 \mathrm{~g} / \mathrm{l}$, and the prothrombin time $14 \mathrm{~s}$ (control $13 \mathrm{~s}$ ); there was no ascites or clinical evidence of hepatic encephalopathy. She was started on propranolol $40 \mathrm{mg}$ twice daily to prevent further variceal bleeding, and her pulse fell from 80 to 62 beats/min. Initially she tolerated propranolol well and was discharged home. Four weeks later, however, she was readmitted with hepatic encephalopathy. She showed typical asterixis, and the mean electroencephalographic frequency (normal $>8.9 \mathrm{~Hz}$ ) had fallen from $7 \mathrm{~Hz}$ five weeks earlier to $5 \mathrm{~Hz}$. There were no focal neurological signs. She was not feverish, and there was no evidence of gastrointestinal haemorrhage. Plasma urea and electrolyte concentrations were normal, and results of liver function tests were unchanged. Blood glucose concentration was $13.3 \mathrm{mmol} / 1(240 \mathrm{mg} / 100 \mathrm{ml}$ ) (normal $2 \cdot 9-4.8 \mathrm{mmol} / 1$ (52-86 mg/ $100 \mathrm{ml})$ ). A midstream urine sample grew $>10^{5}$ Escherichia coli but no pus cells were seen, and treatment with amoxycillin resulted in sterile urine cultures.

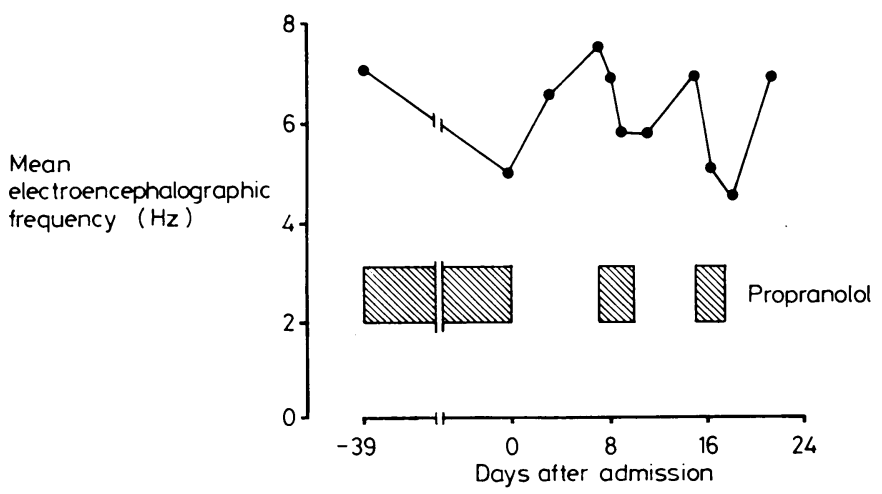

Relation between propranolol treatment and the mean electroencephalographic frequency.

As no obvious cause for the hepatic encephalopathy had been identified propranolol was temporarily stopped in case it was a contributing factor. She was given oral lactulose, magnesium sulphate enemas, and a $40 \mathrm{~g}$ protein diet daily. The hepatic encephalopathy disappeared and the mean electroencephalographic frequency rose to $7.5 \mathrm{~Hz}$. Five days later propranolol $40 \mathrm{mg}$ twice daily was started again. Her pulse fell as before, and within 24 hours she was again encephalopathic and the mean electroencephalographic frequency had fallen to $5 \cdot 7 \mathrm{~Hz}$ (figure). Propranolol was withdrawn and the encephalopathy resolved. A further challenge confirmed that propranolol precipitated hepatic encephalopathy in this patient. No other possible precipitating factors were identified at these times. She remained well over the next seven months.

\section{Comment}

The common causes of acute exacerbation of chronic hepatic encephalopathy are excessive protein intake; gastrointestinal bleeding; constipation; infection; and drugs such as diuretics and sedatives. In our patient the initial episode of encephalopathy was originally thought to have been due to a urinary tract infection. Subsequent challenge with propranolol on two occasions led to rapid worsening of the encephalopathy as assessed clinically and by electroencephalogram. The reduction in liver blood flow caused by propranolol may possibly have impaired liver function sufficiently to induce encephalopathy. Alternatively, a reduction in cerebral blood flow due to propranolol may have been responsible, though there is little evidence that the beta sympathomimetic blockers reduce cerebral blood flow in healthy subjects. ${ }^{3}$

In a short term study of nine patients with cirrhosis given long acting propranolol $160 \mathrm{mg}$ daily Arthur et al showed no consistent deterioration in results of psychometric tests or an increase in serum ammonia concentration and concluded that encephalopathy is not a contraindication to propranolol treatment in patients with cirrhosis and portal hypertension. ${ }^{4} \mathrm{~A}$ significant rise in arterial plasma ammonia concentration, was, however, reported by van Burren et al in six patients with cirrhosis given propranolol $20 \mathrm{mg}$ four times a day. ${ }^{5}$ Encephalopathy was not assessed in their study, but the clear demonstration that propranolol precipitated hepatic encephalopathy in our patient indicates that this is potentially a major complication of propranolol treatment in patients with cirrhosis.

We thank Dr J Kennedy for reporting the electroencephalograms.

${ }^{1}$ Lebrec D, Nouel O, Corbic M, Benhamou J-P. Propranolol-a medical treatment for portal hypertension? Lancet 1980;ii:180-2.

${ }^{2}$ Lebrec D, Poynard T, Hillon P, Benhamou J-P. Propranolol for prevention of recurrent gastrointestinal bleeding in patients with cirrhosis. N Engl f Med 1981;305:1371-4.

${ }^{3}$ Steiner JA, Hughes RJD, James IM. The effect of nadolol on cerebral blood flow. In: Gross F, ed. International experience with nadolol, a long-acting $\beta$-blocking agent. London: Royal Society of Medicine, 1981:99-102.

'Arthur MJP, Tanner AR, Patel C, George CF, Wright R. Portal hypertension, propranolol and hepatic encephalopathy. Lancet 1982;ii:879.

${ }^{5}$ van Burren HR, van der Velden PC, Koorevaar G, Silberbusch J. Propranolol increases arterial ammonia in liver cirrhosis. Lancet 1982;ii: 951-2.

(Accepted 11 May 1983)

Department of Medicine, Royal Free Hospital School of Medicine, London NW3 2PF

D TARVER, $M B$, house physician

R P WALT, MB, MRCP, registrar

A A DUNK, MB, MRCP, registrar

W J JENKINS, MD, MRCP, lecturer

S SHERLOCK, DBE, FRCP, professor

Correspondence to: Dr W J Jenkins.

\section{Non-reducible rotational head tilt in ankylosing spondylitis}

The occurrence of a non-reducible rotational head tilt in $10 \%$ of an unselected group of patients with rheumatoid arthritis has recently been reported. ${ }^{1}$ We report a case in a patient with ankylosing spondylitis to draw attention to this condition.

\section{Case report}

A 57 year old man with a 15 year history of ankylosing spondylitis was admitted with a three month history of intermittent lancinating occipital and neck pains and increasingly restricted movements of his neck. Coincidentally, the pain had become almost constant and extremely severe five days before admission. There was no history of trauma. The most obvious clinical finding, in addition to the spinal deformity typical of ankylosing spondylitis, was a deformity of the neck with tilting of the head to the right and rotation of the chin towards the left (a non reducible rotational head tilt). He was afebrile. Hyperaesthesia was noted over the distribution of the right greater occipital nerve. There was no motor weakness or myelopathy. 
Investigations showed: erythrocyte sedimentation rate $55 \mathrm{~mm}$ in the first $12 \cdot 1 \mathrm{~g} / \mathrm{dl}$; and white cell count $5 \cdot 1 \times 10^{9} / 1 ; \mathrm{IgM}$ rheumatoid factor was absent and HLA B27 antigen was present. Radiology of the spine and pelvis showed changes typical of ankylosing spondylitis. Detailed views of the atlantoaxial region showed rotational subluxation of $\mathrm{C} 1$ relative to $\mathrm{C} 2$ and the occiput. There was apparent collapse of the lateral mass of the atlas on the right side, which was confirmed by tomography and computed tomography. Injected lesion.

Conservative management by immobilising the cervical spine in a moulded collar gave rapid symptomatic relief.

\section{Comment}

Ankylosing spondylitis and rheumatoid arthritis differ in the displacement has been reported in both diseases but less commonly in ankylosing spondylitis. ${ }^{2}{ }^{3} \mathrm{~A}$ recent report of non-reducible rotational head tilt in patients with rheumatoid arthritis attributed this deformity to collapse of the lateral masses of the atlas and axis as a result of erosive arthropathy of the facetal joints. ${ }^{1}$ We report the first case of non-reducible rotational head tilt in ankylosing spondylitis. Special radiological views of the upper cervical spine are required in rheumatoid arthritis. In ankylosing spondylitis radiographs of the cervical spine may be more difficult to interpret. The appearance on the lateral view may be misinterpreted as a fracture of a vertebral facet. Views through the mouth are difficult to obtain in patients with abnormal neck posture. A slight degree of rotation of the head may result in radiographic artefacts mimicking rotatory and lateral subluxations. Tomography of the upper cervical spine was helpful in our case, and computed tomography permitted more precise diagnosis of the subluxations.

The development of non-reducible rotational head tilt appears to have two components. Firstly, there is destruction of part of the lateral mass of the atlas. In rheumatoid arthritis and ankylosing spondylitis this is secondary to chronic synovitis with erosion of cartilage and subchondral bone and eventual destruction and collapse of the lateral mass. Secondly, although in rheumatoid arthritis ligamentous laxity may contribute to the rotational element of the deformity, this is unlikely to be an important feature of ankylosing spondylitis, in which fibrosis and even bony ankylosis are the usual outcome. It might be argued that in ankylosing spondylitis conservative treatment of nonreducible rotational head tilt with immobilisation in a support collar is more likely to succeed because subsequent ankylosis of the interfacetal joints would tend to stabilise the deformity and relieve the pain.

${ }^{1}$ Halla JT, Fallahi S, Hardin JG. Non reducible rotational head tilt and lateral mass collapse. Arthritis Rheum 1982;25:1316-23.

${ }^{2}$ Martel W. The occipito-atlanto-axial joints in rheumatoid arthritis and ankylosing spondylitis. Am $\mathcal{F}$ Roentgenol $1961 ; 86: 223-40$.

3 Sharp J, Purser DW. Spontaneous atlanto-axial dislocation in ankylosing spondylitis and rheumatoid arthritis. Ann Rheum Dis 1961;20:47-76.

- Weinstein PR, Karpman RR, Gall EP, Pitt M. Spinal cord injury, spinal fracture, and spinal stenosis in ankylosing spondylitis. $\mathcal{f}$ Neurosurg 1982; 57:609-16.

(Accepted 12 May 1983)

Department of Rheumatology, Royal Victoria Hospital, Belfast, Northern Ireland

$M$ B FINCH, MB, MRCP, senior registrar

B PAGE, MB, BCH, senior house officer

S D ROBERTS, MD, FRCP, consultant rheumatologist

Correspondence to: Dr M B Finch. hour; $C$ reactive protein concentration normal; haemoglobin concentration white blood cells labelled with isotopes were not taken up at the site of the prevalence and distribution of disease of the spinal joints. Atlantoaxial Subsequent excision of the rib resulted in an immediate cessation of the pain, "renal pain."

\section{Twelfth rib syndrome: a differential diagnosis of loin pain}

The slipping or clicking rib syndrome affecting the eighth, ninth, or 10 th rib is recognised as a cause of upper abdominal pain. ${ }^{1-3}$ Pain associated with the 11th or 12th rib is less well known and has been reported only twice. ${ }^{45}$ We report on four consecutive patients whose pain was in the loin region and was due to intercostal neuralgia associated with the 11 th or 12 th rib, or both, who were cured by resection of the relevant rib or ribs. The patients were followed up for between six and 30 months (mean 17 months).

\section{Case reports}

Case 1-A 34 year old woman complained of intermittent right loin pain over the previous five years. There were no associated symptoms and no history of trauma. The pain was sometimes preceded by certain activities, and, interestingly, she found that using a vacuum cleaner was especially likely to produce discomfort. She had consulted practitioners in various specialties about her pain and had been extensively investigated on several occasions with no result. On examination we found that manipulation of the tip of the right 12th rib exactly and dramatically reproduced her pain. and she remained pain free 30 months after the operation.

Case 2-A 21 year old girl was referred because of persistent left loin pain. She had been extensively investigated previously, when gastrointestinal and repeated urological $x$ ray films had been normal. Urine culture had consistently yielded negative results, and a gynaecological examination had shown no abnormality. Most recently a therapeutic trial with antibiotics had failed to cure her "chronic pyelonephritis." She had complained of severe intermittent pain in her left loin over the previous two years. Initially the pain was described as sharp and radiating towards the corresponding groin, but subsequently, during the attacks, the pain became a dull ache lasting for some 24 hours at a time. She had noticed that she could gain relief by flexion of her spine. Manipulation of the left 11 th and 12th ribs resulted in exquisite tenderness, exactly reproducing her pain. The ribs were resected with immediate relief of her pain, which did not recur during 18 months of follow up.

Case 3-A 37 year old woman complained of an ache in her left loin over the previous 10 months. It tended to be worse at night, when it disturbed her sleep, and was relieved by sitting forwards. There was no history of trauma, and no associated symptoms were present, but she recalled a milder form of the same discomfort during her two pregnancies 17 and eight years previously. Manipulation of the left 11 th and 12 th ribs exactly reproduced her pain, and after excision of the ribs the pain disappeared. She remained pain free in the 15 months after the operation.

Case 4-A 19 year old girl presented with acute right loin pain and a history of intermittent right loin pain for 18 months. The pains were initially sharp but then subsided into a dull ache lasting up to two days. The pain radiated to her right groin and subcostal region and was exacerbated by rotation of the trunk. The symptoms had been attributed to urinary tract infections, but intravenous urography had yielded normal results and infection had been proved on only one occasion. Examination elicited extreme tenderness over the 11 th rib, and manipulation of that rib exactly reproduced her symptoms. The rib was resected and she remained pain free six months after the operation.

\section{Comment}

The hallmark of the syndrome we describe is pain in the loin. This may be intermittent or continuous and may be described as sharp pain or a dull ache or a combination of the two. None of our patients gave a history of trauma to the ribs or relevant associated symptoms. The condition appears to be quite common, and one of us (DGM) has seen four further cases referred for investigation of

The diagnosis is a clinical one and is made when the patient's pain is exactly reproduced by manipulation of the affected rib or ribs. Patients may be referred for investigation of presumed renal pain, and failure to recognise the true cause of the symptoms may lead to unnecessary investigations, possible inappropriate treatment, and needlessly prolonged pain for want of a simple surgical procedure.

${ }^{1}$ Davies-Colley R. Slipping rib. $B r \operatorname{Med} \mathcal{F} 1922$;i:432.

${ }^{2}$ Holmes JF. Slipping rib cartilage. Am $\mathcal{F}$ Surg 1941 ;54:326-38.

${ }^{3}$ Heinz GJ, Zavala DC. Slipping rib syndrome. $\mathcal{F} A M A$ 1977;237:794-5.

"Rawlings MS. The "rib syndrome." Diseases of the chest $1962 ; 41: 432-41$.

${ }^{5}$ Soltau HKV. Memoranda. Br Med F 1922;i:516.

(Accepted 11 May 1983)

Royal Liverpool Hospital, Liverpool L7 8XP

DEREK G MACHIN, FRCs, Merseyside Association for Kidney Research fellow and honorary senior registrar in urology

Arrowe Park Hospital, Wirral, Merseyside L49 5PE

JOHN M SHENNAN, FRCS, FRCSED, consultant surgeon

Correspondence and reprint requests to: Mr D G Machin 